\title{
AN EXTENSION OF KAKUTANI'S THEOREM ON INFINITE PRODUCT MEASURES TO THE TENSOR PRODUCT OF SEMIFINITE $w^{*}$-ALGEBRAS
}

\author{
BY \\ DONALD BURES
}

Suppose that $\left(\mathscr{A}_{i}\right)_{i \in I}$ is a family of semifinite $w^{*}$-algebras and that $\mu_{i}$ is a normal state of $\mathscr{A}_{i}$ with $\mu_{i}(1)=1$ for each $i \in I$. Let $\mathscr{A}=\bigotimes_{i \in I}\left(\mathscr{A}_{i}, \mu_{i}\right)$ and let $A_{i} \rightarrow \bar{A}_{i}$ denote the natural injection of $\mathscr{A}_{i}$ into $\mathscr{A}$. (The notation is explained in $\S 3$ below; $\mathscr{A}$ is the $\left(\mu_{i}\right)$-incomplete direct product of $\left(\mathscr{A}_{i}\right)$ : see [9], [12] or [1].) Given a normal state $\nu_{i}$ of $\mathscr{A}_{i}$ for each $i \in I$, a normal state $\nu$ of $\mathscr{A}$ is written $\bigotimes_{i \in I} \nu_{i}$ when

$$
\nu\left(\prod_{i \in F} \bar{A}_{i}\right)=\prod_{i \in F} \nu_{i}\left(A_{i}\right)
$$

for all $A_{i} \in \mathscr{A}_{i}$ and all finite subsets $F$ of $I$.

Our main result (Theorem 4.1) is that $\bigotimes_{i \in I} \nu_{i}$ exists on $\mathscr{A}$ if and only if $\sum_{i \in I}\left[d\left(\mu_{i}, \nu_{i}\right)\right]^{2}$ converges, or, equivalently, if and only if $\prod_{i \in I} \rho\left(\mu_{i}, \nu_{i}\right)$ converges.

Here $d$ is a metric on the set of normal states of a $w^{*}$-algebra $\mathscr{B} . d$ is defined essentially by $d(\mu, \nu)=\inf \{\|x-y\|\}$, the infimum being taken over all vectors $x$ and $y$ inducing $\mu$ and $\nu$ relative to a representation of $\mathscr{B}$ as a von Neumann algebra. $\rho$ is a kind of inner product defined by

$$
2 \rho(\mu, \nu)=\mu(1)+\nu(1)-[d(\mu, \nu)]^{2} .
$$

We show that $d$ and $\rho$ correspond to Kakutani's $d$ and $\rho$ [6] when $\mathscr{B}$ is abelian (and normal states are made, in the usual fashion, to correspond to measures absolutely continuous with respect to a fixed measure). Thus our result reduces to Kakutani's [6] when each $\mathscr{A}_{i}$ is abelian.

We give two applications of our main result. First, suppose that $\phi_{i}$ is an isomorphism of the $w^{*}$-algebra $\mathscr{A}_{i}$ onto the $w^{*}$-algebra $\mathscr{B}_{i}$. Then we show that an isomorphism $\phi$ from $\otimes\left(\mathscr{A}_{i}, \mu_{i}\right)$ to $\otimes\left(\mathscr{B}_{i}, \nu_{i}\right)$ such that

$$
\phi\left(\bar{A}_{i}\right)=\overline{\phi_{i}\left(A_{i}\right)} \text { for all } A_{i} \in \mathscr{A}_{i} \text { and all } i \in I
$$

exists if and only if

$$
\sum\left[d\left(\mu_{i}, \nu_{i} \circ \phi_{i}\right)\right]^{2}<\infty .
$$

Secondly, we show that if each $\mathscr{A}_{i}$ is a finite factor with normalized normal trace $\tau_{i}$, then $\otimes\left(\mathscr{A}_{i}, \mu_{i}\right)$ is finite if and only if

$$
\sum\left[d\left(\mu_{i}, \tau_{i}\right)\right]^{2}<\infty .
$$

This result generalizes results in [1] and [7].

Received by the editors June 28, 1967. 
Further applications, concerning unitary equivalence of representations of (weak) infinite product groups and unitary equivalence of representations of infinite $c^{*}$-tensor-products, will be discussed elsewhere.

We begin, in $\S 1$, with the definitions and fundamental properties of $d$ and $\rho$ on an arbitrary $w^{*}$-algebra. In $\S 2$ we prove the product formula for $\rho$ on a finite tensor product of semifinite $w^{*}$-algebras. In $\S 3$ we establish our notation for infinite tensor products and summarize some of the properties of the tensor product that we need. $\S 3$ contains no new results. We conclude, in $\S 4$, with the main results and applications.

1. Definition and properties of $\rho$ and $d$. Throughout this section $\mathscr{A}$ will denote a $w^{*}$-algebra and $\Sigma$ will denote the set of normal states of $\mathscr{A}$. By a representation $\phi$ of $\mathscr{A}$ on $H$ we mean an isomorphism of $\mathscr{A}$ onto a von Neumann algebra acting on $H$. (Notice that $\phi(1)$ is necessarily the identity operator on $H$.)

Definition 1.1. Suppose that $\phi$ is a representation of $\mathscr{A}$ on $H$. For each $\mu \in \Sigma$ define $S(\phi, \mu)$ by:

$$
S(\phi, \mu)=\{x \in H:(\phi(A) x \mid x)=\mu(A) \text { for all } A \in \mathscr{A}\} .
$$

We will say that a vector $x \in S(\phi, \mu)$ induces the state $\mu$ of $\mathscr{A}$ relative to $\phi$.

Definition 1.2. Suppose that $\phi$ is a representation of $\mathscr{A}$ and that $\mu$ and $\nu$ are in $\Sigma$. If either $S(\phi, \mu)$ or $S(\phi, \nu)$ is empty define $\rho_{\phi}(\mu, \nu)=0$ and $d_{\phi}(\mu, \nu)$ $=[\mu(1)+\nu(1)]^{1 / 2}$; otherwise define

$$
\begin{aligned}
& \rho_{\phi}(\mu, \nu)=\sup \{|(x \mid y)|: x \in S(\phi, \mu) \text { and } y \in S(\phi, \nu)\}, \\
& d_{\phi}(\mu, \nu)=\inf \{\|x-y\|: x \in S(\phi, \mu) \text { and } y \in S(\phi, \nu)\} .
\end{aligned}
$$

Definition 1.3. For all $\mu, \nu \in \Sigma$ define:

$$
\rho(\mu, \nu)=\sup \left\{\rho_{\phi}(\mu, \nu): \phi \in \Lambda\right\}, \quad d(\mu, \nu)=\inf \left\{d_{\phi}(\mu, \nu): \phi \in \Lambda\right\},
$$

where $\Lambda$ is the set of all representations of $\mathscr{A}$.

LEMMA 1.4. For all $\mu, \nu \in \Sigma$ and all representations $\phi$ of $\mathscr{A}$ :

$$
\begin{gathered}
{\left[d_{\phi}(\mu, \nu)\right]^{2}=\mu(1)+v(1)-2 \rho_{\phi}(\mu, \nu),} \\
{[d(\mu, \nu)]^{2}=\mu(1)+\nu(1)-2 \rho(\mu, \nu) .}
\end{gathered}
$$

Proof. Obvious.

LEMMA 1.5. For all $\mu, \nu \in \Sigma$ and all real $k \geqq 0$ :

$$
\begin{aligned}
0 \leqq d(\mu, \nu) & \leqq[\mu(1)+\nu(1)]^{1 / 2}, & 0 \leqq \rho(\mu, \nu) & \leqq[\mu(1)+\nu(1)]^{1 / 2}, \\
d(\mu, \nu) & =d(\nu, \mu), & \rho(\mu, \nu) & =\rho(\nu, \mu), \\
d(\mu, \mu) & =0, & \rho(\mu, \mu) & =\mu(1), \\
d(k \mu, k \nu) & =k d(\mu, \nu), & \rho(k \mu, \nu) & =k \rho(\mu, \nu) .
\end{aligned}
$$

Proof. Obvious. 
Proposition 1.6. There exists a representation $\phi$ of $\mathscr{A}$ on $H$ such that

(A) For all $\mu, \nu \in \Sigma: d(\mu, \nu)=d_{\phi}(\mu, \nu)$ and $\rho(\mu, \nu)=\rho_{\phi}(\mu, \nu)$.

(B) For a fixed $\mu \in \Sigma$, there exists $x_{0} \in S(\phi, \mu)$ such that

$$
d(\mu, \nu)=\inf \left\{\left\|x_{0}-y\right\|: y \in S(\phi, \nu)\right\} \text { for all } \nu \in \Sigma .
$$

(C) Suppose that $E$ is a projection of $\mathscr{A}$ and that $\phi^{\prime}$ is the representation of $\mathscr{A}_{E}$ taking EAE into the restriction of $\phi(E A E)$ to $\phi(E) H$. (We call $\phi^{\prime}$ the restriction of $\phi$ to $\mathscr{A}_{E}$.) Then, for all normal states $\mu^{\prime}$ and $\nu^{\prime}$ of $\mathscr{A}_{E}, d\left(\mu^{\prime}, \nu^{\prime}\right)=d_{\phi^{\prime}}\left(\mu^{\prime}, \nu^{\prime}\right)$ and $\rho\left(\mu^{\prime}, \nu^{\prime}\right)$ $=\rho_{\phi^{\prime}}\left(\mu^{\prime}, \nu^{\prime}\right)$.

Proof. It is well known that there exists a representation $\phi_{1}: A \rightarrow A_{1}$ of $\mathscr{A}$ on $H_{1}$ with the property that $S\left(\phi_{1}, \mu\right)$ is nonempty for all $\mu \in \Sigma$. Furthermore it is easy to see that the restriction $\phi_{1}^{\prime}$ of $\phi_{1}$ to $\mathscr{A}_{E}$ will also have this property. Let $\phi$ be the representation

$$
\phi_{1} \oplus \phi_{1}: A \rightarrow A_{1} \oplus A_{1}
$$

of $\mathscr{A}$ on the Hilbert space $H=H_{1} \oplus H_{1}$. Evidently the restriction $\phi^{\prime}$ of $\phi$ to $\mathscr{A}_{E}$ equals $\phi_{1}^{\prime} \oplus \phi_{1}^{\prime}$. Therefore, if we can prove that (A) holds for $\phi$ of the above form, (C) will follow.'

Let us proceed with the proof of (A). Let $\mu$ and $\nu$ be in $\Sigma$, let $\phi_{2}: A \rightarrow A_{2}$ be a representation of $\mathscr{A}$ on $H_{2}$ and let $x_{2} \in S\left(\phi_{2}, \mu\right)$ and $y_{2} \in S\left(\phi_{2}, \nu\right)$. To prove (A), it suffices to demonstrate the existence of vectors $x$ and $y$ in $H$, with $x \in S(\phi, \mu)$ and $y \in S(\phi, \nu)$, such that

$$
\|x-y\| \leqq\left\|x_{2}-y_{2}\right\|
$$

Let $x_{1}$ be in $S(\phi, \mu)$. Then

$$
\psi: A_{1} x_{1} \rightarrow A_{2} x_{2} \text { for all } A \in \mathscr{A}
$$

is an isometry which extends to an isometry $\psi$ from $\left[\mathscr{A}_{1} x_{1}\right]$ onto $\left[\mathscr{A}_{2} x_{2}\right]$. Let $E_{2}$ be the orthogonal projection of $H_{2}$ onto $\left[\mathscr{A}_{2} x_{2}\right]$; evidently $E_{2}$ commutes with $\mathscr{A}_{2}$. Let $y_{2}^{\prime}=E_{2} y_{2}$ and $y_{2}^{\prime \prime}=y_{2}-y_{2}^{\prime}$. Then, relative to $\phi_{2}, y_{2}^{\prime}$ induces $\nu^{\prime}$ and $y_{2}^{\prime \prime}$ induces $\nu^{\prime \prime}$ with $\nu=\nu^{\prime}+\nu^{\prime \prime}$. Let $y_{1}^{\prime}=\psi^{-1}\left(y_{2}^{\prime}\right)$; then $y_{1}^{\prime}$ induces $\nu^{\prime}$ relative to $\phi_{1}$ because $\psi$ is interlacing. Let $y^{\prime \prime}$ be in $S\left(\phi_{2}, \nu^{\prime \prime}\right)$. Take $x=x_{1} \oplus 0$ and $y=y_{1}^{\prime} \oplus y_{1}^{\prime \prime}$. Then we have $x \in S(\phi, \mu)$ and $y \in S(\phi, \nu)$, and furthermore

$$
\begin{aligned}
\|x-y\|^{2} & =\left\|x_{1}-y_{1}^{\prime}\right\|^{2}+\left\|y_{1}^{\prime \prime}\right\|^{2} \\
& =\left\|\psi\left(x_{1}\right)-\psi\left(y_{1}^{\prime}\right)\right\|^{2}+\left\|y_{2}^{\prime \prime}\right\|^{2} \\
& =\left\|x_{2}-y_{2}^{\prime}\right\|^{2}+\left\|y_{2}^{\prime \prime}\right\|^{2}=\left\|x_{2}-y_{2}\right\|^{2} .
\end{aligned}
$$

That demonstrates (1.1) and completes the proof of (A).

(B) follows immediately from the observation that $x=x_{1} \oplus 0$ was chosen independently of $\nu$ and $\phi_{2}$.

Proposition 1.7. $d:(\mu, \nu) \rightarrow d(\mu, \nu)$ is a metric on $\Sigma$. 
Proof. Let $\mu$ be given in $\Sigma$. Take the $\phi$ and $x_{0} \in S(\phi, \mu)$ of Proposition 1.6.

Then $d(\mu, \nu)=0$ implies

$$
\inf \left\{\left\|x_{0}-y\right\|: y \in S(\phi, \nu)\right\}=0 .
$$

Since $S(\phi, \nu)$ is clearly a closed subset of $H,(1.2)$ implies that $x_{0}$ is in $S(\phi, \nu)$ or that $\mu=\nu$. We have shown that $d(\mu, \nu)=0$ implies $\mu=\nu$.

To prove the triangle inequality, suppose that $\nu$ and $\omega$ are in $\Sigma$. Then:

$$
\begin{aligned}
d(\nu, \omega) & =\inf \{\|y-z\|: y \in S(\phi, \nu), z \in S(\phi, \omega)\} \\
& \leqq \inf \left\{\left\|y-x_{0}\right\|+\left\|x_{0}-z\right\|\right\}=d(\nu, \mu)+d(\mu, \omega) .
\end{aligned}
$$

Proposition 1.8. (A) For all $\mu, \nu \in \Sigma, d(\mu, \mu+\nu) \leqq(\nu(1))^{1 / 2}$.

(B) For all $\mu, \nu \in \Sigma$ with $\mu(1), \nu(1) \leqq 1$ and for $0 \leqq \varepsilon \leqq 1$,

$$
|d(\mu, \nu)-d(\mu,(1-\varepsilon) \nu+\varepsilon \mu)| \leqq 2 \varepsilon^{1 / 2} .
$$

Proof. (A) Let $\phi_{1}: A \rightarrow A_{1}$ be a representation of $\mathscr{A}$ on $H_{1}$ such that vectors $x_{1}$ and $y_{1}$ exist with $x_{1} \in S\left(\phi_{1}, \mu\right)$ and $y_{1} \in S\left(\phi_{1}, \nu\right)$. Let $\phi$ be $\phi_{1} \oplus \phi_{1}$. Then $x=x_{1}$ $\oplus 0 \in S(\phi, \mu)$ and $z=x_{1} \oplus y_{1} \in S(\phi, \mu+\nu)$. Therefore

$$
d(\mu+\nu, \mu) \leqq\|z-x\|=\left\|y_{1}\right\|=(\nu(1))^{1 / 2} .
$$

(B) Using the triangle inequality for $d$ and (A), we obtain:

$$
\begin{aligned}
|d(\mu, \nu)-d(\mu,(1-\varepsilon) \nu+\varepsilon \mu)| & \leqq d(\nu,(1-\varepsilon) \nu+\varepsilon \mu) \\
& \leqq d(\nu,(1-\varepsilon) \nu)+d((1-\varepsilon) \nu,(1-\varepsilon) \nu+\varepsilon \mu) \\
& \leqq(\varepsilon \nu(1))^{1 / 2}+(\varepsilon \mu(1))^{1 / 2} \leqq 2 \varepsilon^{1 / 2} .
\end{aligned}
$$

Proposition 1.9. (A) For $\mu, \nu \in \Sigma$ with $\mu(1), \nu(1) \leqq 1,|\mu(1)-\nu(1)| \leqq 2 d(\mu, \nu)$.

(B) Suppose that $\mu, \mu^{\prime}, \nu, \nu^{\prime} \in \Sigma$ with $\mu(1), \mu^{\prime}(1), \nu(1), \nu^{\prime}(1) \leqq 1$, that $\varepsilon>0$ and that $d\left(\mu, \mu^{\prime}\right)<\varepsilon$ and $d\left(\nu, \nu^{\prime}\right)<\varepsilon$. Then $\left|\rho(\mu, \nu)-\rho\left(\mu^{\prime}, \nu^{\prime}\right)\right|<5 \varepsilon$.

(C) Suppose that $\mu, \nu \in \Sigma$ with $\mu(1)=\nu(1)=1$, and that $P$ and $Q$ are projections of $\mathscr{A}$ with $\mu(P)>1-\varepsilon$ and $\nu(Q)>1-\varepsilon$. Then $\left|\rho(\mu, \nu)-\rho\left(\mu_{P}, \nu_{Q}\right)\right|<5 \varepsilon^{1 / 2}$.

Proof. (A) Suppose that $x$ induces $\mu$ and $y$ induces $\nu$. Then

$$
\begin{aligned}
|\mu(1)-v(1)| & =\left|\|x\|^{2}-\|y\|^{2}\right| \\
& =(\|x\|+\|y\|)|\|x\|-\|y\|| \leqq 2\|x-y\| .
\end{aligned}
$$

Since $d(\mu, \nu)$ is the infimum of such $\|x-y\|$, (A) follows.

(B) Using Lemma 1.4, the triangle inequality for $d$, and (A), we obtain:

$$
\begin{aligned}
2\left|\rho(\mu, \nu)-\rho\left(\mu, \nu^{\prime}\right)\right| & =\left|\nu(1)-\nu^{\prime}(1)+\left(d\left(\mu, \nu^{\prime}\right)\right)^{2}-(d(\mu, \nu))^{2}\right| \\
& \leqq\left|\nu(1)-\nu^{\prime}(1)\right|+\left(d\left(\mu, \nu^{\prime}\right)+d(\mu, \nu)\right)\left|d\left(\mu, \nu^{\prime}\right)-d(\mu, \nu)\right| \\
& \leqq 2 d\left(\nu, \nu^{\prime}\right)+2 \sqrt{ } 2 d\left(\nu, \nu^{\prime}\right)<5 \varepsilon .
\end{aligned}
$$

Similarly $2\left|\rho\left(\mu, \nu^{\prime}\right)-\rho\left(\mu^{\prime}, \nu^{\prime}\right)\right|<5 \varepsilon$ and (B) follows.

(C) We obtain from (A) of Proposition 1.8 that

$$
d\left(\mu, \mu_{P}\right)=d\left(\mu_{P}+\left(\mu-\mu_{P}\right), \mu_{P}\right) \leqq\left[\left(\mu-\mu_{P}\right)(1)\right]^{1 / 2}<\varepsilon^{1 / 2}
$$

and similarly $d\left(\nu, \nu_{Q}\right)<\varepsilon^{1 / 2}$.

Hence $(C)$ is a consequence of $(B)$. 
Proposition 1.10. Suppose that $\mu$ and $\nu$ are normal states of $\mathscr{A}$, and that $E$ is a projection of $\mathscr{A}$ with $\mu(E)=\nu(E)=1$.

Let $\mu^{\prime}$ and $\nu^{\prime}$ denote the restrictions of $\mu$ and $\nu$ to $\mathscr{A}_{E}$. Then $\rho(\mu, \nu)=\rho\left(\mu^{\prime}, \nu^{\prime}\right)$ and $d(\mu, \nu)=d\left(\mu^{\prime}, \nu^{\prime}\right)$.

Proof. Let $\phi$ be a representation of $\mathscr{A}$ on $H$ such that the conditions of Proposition 1.6 hold. Let $\phi^{\prime}$ be the restriction (in the sense of Proposition 1.6) of $\phi$ to $\mathscr{A}_{E}$. It is easy to confirm that $S(\phi, \mu)=S\left(\phi^{\prime}, \mu^{\prime}\right)$ and $S(\phi, \nu)=S\left(\phi^{\prime}, \nu^{\prime}\right)$. From here (A) and $(\mathrm{C})$ of Proposition 1.6 complete the proof.

Definition 1.11. Suppose that $\mu$ and $\nu$ are in $\Sigma_{1}=\{\mu \in \Sigma: \mu(1)=1\}$. Define

$$
\delta(\mu, \nu)=2 \sup \{|\mu(E)-\nu(E)|: E \text { a projection of } \mathscr{A}\} .
$$

RemarK. Evidently $\delta(\mu, \nu) \leqq\|\mu-v\|$, the uniform norm of the functional $\mu-\nu$ on $\mathscr{A}$. In fact it is easy to see that

$$
\delta(\mu, \nu)=\sup \left\{|(\mu-\nu)(A)|: A \in \mathscr{A} \text { with } A=A^{*} \text { and }\|A\| \leqq 1\right\} .
$$

From here we can conclude that $\delta(\mu, \nu)=\|\mu-\nu\|$ (see [5] or [3, 2.6.4]). For our purposes here Definition 1.11 is the more suitable.

Proposition 1.12. For all $\mu, \nu \in \Sigma_{1}$

$$
[d(\mu, \nu)]^{2} \leqq \delta(\mu, \nu) .
$$

Proof. It is sufficient to find a projection $E$ of $\mathscr{A}$, a representation $\phi$ of $\mathscr{A}$, and vectors $x \in S(\phi, \mu)$ and $y \in S(\phi, \nu)$ such that

$$
\|x-y\|^{2} \leqq 2|\mu(E)-\nu(E)| .
$$

Let us suppose, at first, that $\nu \leqq n \mu$ for some integer $n$. Then, by Sakai's RadonNikodym theorem [10], $\nu=\mu_{T}$ for some $T \in \mathscr{A}^{+}$. Let $\left(E_{\lambda}\right)$ be the spectral resolution of $T$ and take $E=E_{1}$. Let $\phi$ be a representation of $\mathscr{A}$ such that $S(\phi, \mu)$ is nonempty and take $x \in S(\phi, \mu)$. Take $y=\phi(T) x$; evidently $y \in S(\phi, \nu)$. Then, writing $T$ for $\phi(T)$ and $E$ for $\phi(E)$, we obtain:

$$
\begin{aligned}
\|x-y\|^{2} & =\|x-T x\|^{2}=\left((1-T)^{2} E x \mid x\right)+\left((T-1)^{2}(1-E) x \mid x\right) \\
& \leqq((1-T)(1+T) E x \mid x)+((T-1)(T+1)(1-E) x \mid x) \\
& =[(E x \mid x)-((1-E) x \mid x)]+[((1-E) T x \mid T x)-(E T x \mid T x)]=2[\mu(E)-\nu(E)] .
\end{aligned}
$$

Thus (1.4) holds and from there (1.3) holds, whenever $\nu \leqq n \mu$ for some integer $n$.

Suppose now that $\mu$ and $\nu$ are arbitrary in $\Sigma_{1}$. For $0<\varepsilon<1$ let $\mu^{\prime}=(1-\varepsilon) \mu+\varepsilon \nu$. Then $\nu \leqq n \mu^{\prime}$ for $n \geqq 1 / \varepsilon$, so that, by the preceding paragraph,

$$
\left[d\left(\mu^{\prime}, v\right)\right]^{2} \leqq \delta\left(\mu^{\prime}, \nu\right) .
$$

Clearly

$$
\delta\left(\mu^{\prime}, \nu\right)=(1-\varepsilon) \delta(\mu, \nu) .
$$


By Proposition $1.8(\mathrm{~B})$

$$
\left|d\left(\mu^{\prime}, \nu\right)-d(\mu, \nu)\right| \leqq 2 \varepsilon^{1 / 2} .
$$

Combining (1.5), (1.6) and (1.7), we obtain

$$
d(\mu, \nu) \leqq 2 \varepsilon^{1 / 2}+[\delta(\mu, \nu)]^{1 / 2} .
$$

Since $\varepsilon$ is arbitrary with $0<\varepsilon<1$, we can conclude that $\mu$ and $\nu$ satisfy (1.3).

Corollary 1.13. Suppose that $\mu, \nu \in \Sigma_{1}$ and $\varepsilon>0$. Then $\rho(\mu, \nu)<\varepsilon$ implies that there exists a projection $E$ of $\mathscr{A}$ such that:

$$
\mu(E)>1-\varepsilon \text { and } \nu(E)<\varepsilon .
$$

Proof. Suppose that $\rho(\mu, \nu)<\varepsilon$. Then $[d(\mu, \nu)]^{2}>2(1-\varepsilon)$ and, by Proposition $1.12, \delta(\mu, \nu)>2(1-\varepsilon)$.

The definition of $\delta$ shows now that there exists a projection $P$ of $\mathscr{A}$ such that

$$
|\mu(P)-\nu(P)|>1-\varepsilon .
$$

Take $E=P$ if $\mu(P)>\nu(P)$ and $E=1-P$ if $\mu(P)<\nu(P)$. Then (1.8) is a consequence of (1.9).

2. The product formula for $\rho$. In this section, we are concerned primarily with establishing the formula

$$
\rho\left(\mu_{1} \otimes \mu_{2}, \nu_{1} \otimes \nu_{2}\right)=\left[\rho\left(\mu_{1}, \mu_{2}\right)\right]\left[\rho\left(\nu_{1}, \nu_{2}\right)\right]
$$

for normal states $\mu_{1}$ and $\nu_{1}$ of $\mathscr{A}_{1}$ and $\mu_{2}$ and $\nu_{2}$ of $\mathscr{A}_{2}$, where $\mathscr{A}_{1}$ and $\mathscr{A}_{2}$ are semifinite $w^{*}$-algebras. Here $\mu_{1} \otimes \mu_{2}$ denotes the normal state of $\mathscr{A}_{1} \otimes \mathscr{A}_{2}$ defined by

$$
\left(\mu_{1} \otimes \mu_{2}\right)\left(A_{1} \otimes A_{2}\right)=\left[\mu_{1}\left(A_{1}\right)\right]\left[\mu_{2}\left(A_{2}\right)\right] \text { for all } A_{1} \in \mathscr{A}_{1} \text { and all } A_{2} \in \mathscr{A}_{2} \text {. }
$$

If $\mu$ is a state of $\mathscr{A}$ and $M \in \mathscr{A}$, we follow the standard usage in defining the state $\mu_{M}$ of $\mathscr{A}$ by $\mu_{M}(A)=\mu\left(M^{*} A M\right)$ for all $A \in \mathscr{A}$.

LEMMA 2.1. Suppose that $\phi$ is a representation of the $w^{*}$-algebra $\mathscr{A}$ on $H$ and that $x$ and $y$ in $H$ induce the same state $\mu$ relative to $\phi$. Then there exists a partial isometry $U^{\prime}$ in $(\phi(\mathscr{A}))^{\prime}$ such that $U^{\prime} x=y$.

Proof. A standard result (see [2] or the proof of Proposition 1.6).

LEMMA 2.2. Suppose that $\phi$ is a representation of $\mathscr{A}$ and that the vector $z$ induces a trace relative to $\phi$. Then if $T \in \mathscr{A}^{+}$and $U$ is a unitary operator of $\mathscr{A}, \phi(T U) z$ and $\phi(T) z$ induce the same state relative to $\phi$.

Proof. Obvious by direct calculation.

Proposition 2.3. Suppose that $\tau$ is a normal finite trace on the $w^{*}$-algebra $\mathscr{A}$ and that $M$ and $N$ are in $\mathscr{A}^{+}$. Then $\rho\left(\tau_{M}, \tau_{N}\right)=\tau|M N|$. 
Proof. By assertion 1 of Lemma 1.9 we may, without loss of generality, assume that $\tau$ is faithful. Then $\mathscr{A}$ is finite, so that the polar decomposition of $M N$ yields a unitary operator $U$ of $\mathscr{A}$ such that

$$
M N=U|M N| \text { and } N M U=|M N| .
$$

Denote $\tau_{M}$ by $\mu$ and $\tau_{N}$ by $\nu$. Let $\phi$ be a representation of $\mathscr{A}$ such that $\tau$ is induced by a vector $z$ and

$$
\rho(\mu, \nu)=\rho_{\phi}(\mu, \nu) .
$$

(Such a $\phi$ exists by Proposition 1.6.) Then $\phi(M) z$ induces $\mu, \phi(N) z$ induces $\nu$, and $\phi(M U) z$ also induces $\mu$ (Lemma 2.2). Therefore, using (2.2), we obtain

$$
\rho(\mu, \nu) \geqq|(\phi(M U) z \mid \phi(N) z)|=|\tau(N M U)|=\tau|M N| .
$$

To prove the opposite inequality, let $\phi$ and $z$ be as above and suppose that $x$ induces $\mu$ and $y$ induces $\nu$. Then (Lemma 2.1) there exist partial isometries $U^{\prime}$ and $V^{\prime}$ of $(\phi(\mathscr{A}))^{\prime}$ such that $x=U^{\prime} \phi(M) z$ and $y=V^{\prime} \phi(N) z$. Hence, denoting $|M N|$ by $P$ and using (2.2), we obtain:

$$
\begin{aligned}
|(x \mid y)| & =\left|\left(V^{\prime *} U^{\prime} z \mid \phi(M N) z\right)\right| \\
& =\left|\left(V^{\prime *} U^{\prime} z \mid \phi\left(U P^{1 / 2} P^{1 / 2}\right) z\right)\right|=\left|\left(V^{\prime *} U^{\prime} \phi\left(P^{1 / 2} U\right) z \mid \phi\left(P^{1 / 2}\right) z\right)\right| \\
& \leqq\left\|\phi\left(P^{1 / 2} U\right) z\right\|\left\|\phi\left(P^{1 / 2}\right) z\right\|=\left[\tau\left(U^{*} P U\right)\right]^{1 / 2}[\tau(P)]^{1 / 2}=\tau(P)=|M N| .
\end{aligned}
$$

Since $\rho_{\phi}(\mu, \nu)$ is the supremum of such $|(x \mid y)|$, we obtain from $(2.3) \rho(\mu, \nu)=\rho_{\phi}(\mu, \nu)$ $\leqq \tau|M N|$.

Lemma 2.4. Suppose that $\mathscr{A}$ is a $w^{*}$-algebra and that $\tau$ is a faithful normal semifinite trace on $\mathscr{A}$. Let $\mu$ and $\nu$ be normal states of $\mathscr{A}$ with $\mu(1)=\nu(1)=1$, and let $\varepsilon>0$. Then there exist projections $E, P$ and $Q$ in $\mathscr{A}$ with $P, Q \leqq E$ such that:

1. $\tau(E)<\infty$,

2. $\mu(P)>1-\varepsilon$ and $\nu(Q)>1-\varepsilon$,

3. $\mu_{P}, \nu_{Q} \leqq K \tau_{E}$ for some number $K$.

Proof. Since $\tau$ is semifinite there exists a family $\left(E_{i}\right)$ of projections of $\mathscr{A}$ such that each $\tau\left(E_{i}\right)<\infty$ and $\sum E_{i}=1$. Evidently a suitable finite sum $E$ of $E_{i}$ 's will satisfy $\tau(E)<\infty, \mu(E)>1-\varepsilon$ and $\nu(E)>1-\varepsilon$. Since $\tau$ is faithful, $\mu_{E}$ and $\nu_{E}$ are absolutely continuous with respect to $\tau_{E}$. From there, a weak version of the Radon-Nikodym theorem in $w^{*}$-algebras $[8$, p. 211$]$ tells us that there exist projections $P$ and $Q \leqq E$ and a number $K$ such that 2 and 3 hold.

TheOREM 2.5. Suppose that $\mathscr{A}_{1}$ and $\mathscr{A}_{2}$ are semifinite $w^{*}$-algebras. Then the product formula (2.1) holds.

Proof. Let $\delta=1$ or 2 . We may assume without loss of generality that $\mu_{\delta}(1)$ $=v_{\delta}(1)=1$.

Let $\varepsilon$ be given $>0$. Since $\mathscr{A}_{\delta}$ is semifinite, there exists a faithful normal semifinite trace $\tau_{\delta}$ on $\mathscr{A}_{\delta}$. Choose $E_{\delta}, P_{\delta}$ and $Q_{\delta}$ to satisfy the conditions of Lemma 2.4. 
Denote by $\mu_{\delta}^{\prime}$ and $\nu_{\delta}^{\prime}$ respectively the restrictions of $\left(\mu_{\delta}\right)_{P_{\delta}}$ and $\left(\nu_{\delta}\right)_{Q_{\delta}}$ to $\left(\mathscr{A}_{\delta}\right)_{E_{\delta}}$. By Proposition 1.10 and (C) of Proposition 1.9

$$
\left|\rho\left(\mu_{\delta}, \nu_{\delta}\right)-\rho\left(\mu_{\delta}^{\prime}, \nu_{\delta}^{\prime}\right)\right|<5 \varepsilon^{1 / 2} \text { for } \delta=1 \text { and } 2 .
$$

We note that, if $\tau_{\delta}^{\prime}$ denotes the restriction of $\left(\tau_{\delta}\right)_{E_{\delta}}$ to $\left(\mathscr{A}_{\delta}\right)_{E_{\delta}}$, then $\tau_{\delta}^{\prime}$ is a finite faithful trace on $\left(\mathscr{A}_{\delta}\right)_{E_{\delta}}$, and $\mu_{\delta}^{\prime}, \nu_{\delta}^{\prime} \leqq K \tau_{\delta}^{\prime}$ for $\delta=1$ and 2 .

Now let $\mathscr{A}=\mathscr{A}_{1} \otimes \mathscr{A}_{2}, \mu=\mu_{1} \otimes \mu_{2}, \nu=\nu_{1} \otimes \nu_{2}, E=E_{1} \otimes E_{2}, P=P_{1} \otimes P_{2}$ and $Q=Q_{1} \otimes Q_{2}$. Identify $\mathscr{A}_{E}$ and $\left(\mathscr{A}_{1}\right)_{E_{1}} \otimes\left(\mathscr{A}_{2}\right)_{E_{2}}$ in the canonical way. The restriction $\mu^{\prime}$ of $\mu_{P}$ to $\mathscr{A}_{E}$ evidently equals $\mu_{1}^{\prime} \otimes \mu_{2}^{\prime}$; similarly $\nu^{\prime}=\nu_{1}^{\prime} \otimes \nu_{2}^{\prime}$ where $\nu^{\prime}$ denotes the restriction of $\nu_{Q}$ to $\mathscr{A}_{E}$. We have

$$
\mu(P)=\left(\mu_{1}\left(P_{1}\right)\right)\left(\mu_{2}\left(P_{2}\right)\right)>(1-\varepsilon)^{2}>1-2 \varepsilon
$$

and $\nu(Q)>1-2 \varepsilon$. Therefore, using Proposition 1.10 and $(\mathrm{C})$ of Proposition 1.9, we obtain

$$
\left|\rho(\mu, \nu)-\rho\left(\mu^{\prime}, \nu^{\prime}\right)\right|<5(2 \varepsilon)^{1 / 2} .
$$

Combining (2.4) and (2.5), we see that, since $\varepsilon>0$ is arbitrary, if the product formula (2.1) holds for $\mu_{\delta}^{\prime}$ and $\nu_{\delta}^{\prime}$ then it holds for $\mu_{\delta}$ and $\nu_{\delta}$ also. The proof of Theorem 2.5 can be completed, then, by proving the product formula under the following conditions:

1. For $\delta=1$ and $2, \mathscr{A}_{\delta}$ has a faithful finite normal trace $\tau_{\delta}$.

2. A number $K$ exists such that $\mu_{\delta}$ and $v_{\delta}$ are $\leqq K \tau_{\delta}$ for $\delta=1$ and 2 .

Suppose then that these conditions hold. By a well-known Radon-Nikodym type theorem [2, p. 91], there exist operators $M_{\delta}$ and $N_{\delta}$ of $\mathscr{A}^{+}$such that $\mu_{\delta}=\left(\tau_{\delta}\right)_{M_{\delta}}$ and $v_{\delta}=\left(\tau_{\delta}\right)_{N_{\delta}}$. Then Proposition 2.3 shows that

$$
\rho\left(\mu_{\delta}, \nu_{\delta}\right)=\tau_{\delta}\left|M_{\delta} N_{\delta}\right|
$$

Now it is well known that $\tau=\tau_{1} \otimes \tau_{2}$ defines a finite normal trace on $\mathscr{A}=\mathscr{A}_{1} \otimes \mathscr{A}_{2}$. A direct calculation proves that, if $M=M_{1} \otimes M_{2}$ and $N=N_{1} \otimes N_{2}$, then $\mu_{1} \otimes \mu_{2}$ $=\tau_{M}$ and $\nu_{1} \otimes v_{2}=\tau_{N}$. Again Proposition 2.3 applies to give

$$
\rho\left(\mu_{1} \otimes \mu_{2}, \nu_{1} \otimes \nu_{2}\right)=\tau|M N| \text {. }
$$

A direct calculation shows that

$$
|M N|=\left|M_{1} N_{1}\right| \otimes\left|M_{2} N_{2}\right|
$$

so that

$$
\tau|M N|=\left(\tau_{1}\left|M_{1} N_{1}\right|\right)\left(\tau_{2}\left|M_{2} N_{2}\right|\right) .
$$

(2.6), (2.7) and (2.8) taken together prove the product formula under conditions 1 and 2 . This completes the proof.

COROLlaRY 2.6. Suppose that $\left(\mathscr{A}_{\alpha}\right)_{\alpha \in F}$ is a finite family of semifinite $w^{*}$-algebras. 
Suppose that $\mu_{\alpha}$ and $v_{\alpha}$ are normal states of $\mathscr{A}_{\alpha}$ for each $\alpha \in F$. Then the product formula holds; that is

$$
\rho\left(\bigotimes_{\alpha \in F} \mu_{\alpha}, \bigotimes_{\alpha \in F} \nu_{\alpha}\right)=\prod_{\alpha \in F} \rho\left(\mu_{\alpha}, \nu_{\alpha}\right) .
$$

Proof. This is obtained from Theorem 2.5 by induction and the fact that the tensor product of two semifinite $w^{*}$-algebras is semifinite [2].

REMARK. A direct proof could be given by complicating the proof of Theorem 2.5.

Proposition 2.7. Suppose that $\mathscr{S}$ is a $\sigma$-algebra of subsets of $X$ and that $\mu$ and $\nu$ are finite measures on $\mathscr{S}$. Let $\lambda$ be a $\sigma$-finite measure on $\mathscr{S}$ such that $\mu$ and $\nu$ are absolutely continuous with respect to $\lambda$, and let $\mathscr{A}$ be the $w^{*}$-algebra $L_{\infty}(X, \mathscr{S}, \lambda)$. Then integration with respect to $\mu$ and $\nu$ defines two normal states of $\mathscr{A}$ which we denote by $\mu^{\prime}$ and $\nu^{\prime}$ respectively. Then Kakutani's $\rho(\mu, \nu)$ and $d(\mu, \nu)$ [6] are identical with our $\rho\left(\mu^{\prime}, \nu^{\prime}\right)$ and $d\left(\mu^{\prime}, \nu^{\prime}\right)$.

Proof. Take $\omega=\mu+\nu$ and $\omega^{\prime}=\mu^{\prime}+\nu^{\prime}$, and compare (6) and (7) of [6] with Proposition 2.3 above. Bear in mind that if $\mu^{\prime}=\left(\omega^{\prime}\right)_{M}$ and $M \geqq 0$ then $M$ is the square-root of the Radon-Nikodym derivative of $\mu$ with respect to $\omega$.

REMARK. The formula $\rho\left(\tau_{M}, \tau_{N}\right)=\tau|M N|$ can be shown to hold for $\tau$ a normal semifinite trace and $M$ and $N$ measurable hyperhermitian operators affiliated with $\mathscr{A}$ (see [11] for the terminology and interpretation of $|M N|$ ). Hence, by suitable Radon-Nikodym theorems ([4] and [11]), this formula could be used to define $\rho$ on semifinite $w^{*}$-algebras.

For $\mathscr{A}$ a factor of type I, the situation is much simpler. Let tr denote the HilbertSchmidt trace on $\mathscr{A}$. Then it is easy to see, by a direct calculation, that every normal state $\mu$ of $\mathscr{A}$ is of the form $\operatorname{tr}_{M}$ for $M \geqq 0$ a bounded operator which is Hilbert-Schmidt. It can be shown fairly easily (without appeal to the techniques of [11]), that $\rho\left(\operatorname{tr}_{M}, \operatorname{tr}_{N}\right)=\operatorname{tr}|M N|$. Notice that here $|M N|$ is defined in the usual fashion, since $M$ and $N$ are bounded.

3. Infinite tensor products: notation. Z. Takeda, using inductive limits, has given an algebraic definition of the infinite direct product of a family $w^{*}$-algebras [12]. It is more convenient here for us to represent the $w^{*}$-algebras as von Neumann algebras and to make use of von Neumann's definition of the infinite direct product of Hilbert spaces [9]. For a further discussion of the definitions below, and for proofs of cited results not in [9], see [1].

Let $I$ be an arbitrary indexing set. Suppose that $\left(H_{i}\right)_{i \in I}$ is a family of Hilbert spaces and that for each $i \in I, x_{i} \in H_{i}$ with $\left\|x_{i}\right\|=1$. Then we denote by $\bigotimes_{i \in I}\left(H_{i}, x_{i}\right)$ von Neumann's incomplete direct product of the family $\left(H_{i}\right)$ with respect to the $C_{0}$-sequence $\left(x_{i}\right)$; we call $H=\bigotimes_{i \in I}\left(H_{i}, x_{i}\right)$ the tensor product of $\left(H_{i}\right)$ with respect to $\left(x_{i}\right)$. Let

$$
\Gamma=\left\{\left(y_{i}\right): \text { each } y_{i} \in H_{i} \text { and } \sum\left|1-\left(x_{i} \mid y_{i}\right)\right|+\sum\left|1-\left\|y_{i}\right\|\right|<\infty\right\} .
$$


Then there is a canonical multilinear mapping $\left(y_{i}\right) \rightarrow \bigotimes y_{i}$ from $\Gamma$ into a dense subset of $H$ with

$$
\left(\otimes y_{i} \mid \otimes z_{i}\right)=\prod\left(y_{i} \mid z_{i}\right) \text { for all }\left(y_{i}\right),\left(z_{i}\right) \in \Gamma .
$$

We state the following for reference:

Lemma 3.1. Suppose that $x_{i}, y_{i} \in H_{i}$ with $\left\|x_{i}\right\|=\left\|y_{i}\right\|=1$ and $\sum\left|1-\left(x_{i} \mid y_{i}\right)\right|<\infty$. Then $\otimes\left(H_{i}, x_{i}\right)=\otimes\left(H_{i}, y_{i}\right)$.

Let $H=\bigotimes\left(H_{i}, x_{i}\right)$. Then there exist canonical isomorphisms $A_{i} \rightarrow \bar{A}_{i}$. from $\mathscr{L}\left(H_{i}\right)$ into $\mathscr{L}(H)$. We have $\bar{A}_{k}\left(\otimes y_{i}\right)=\otimes y_{i}^{\prime}$ where $y_{k}^{\prime}=A_{k} y_{k}$ and $y_{i}^{\prime}=y_{i}$ for $i \neq k$. Suppose that $\mathscr{A}_{i}$ is a von Neumann algebra on $H_{i}$. We define the tensor product of the family $\left(\mathscr{A}_{i}\right)$ with respect to $\left(x_{i}\right), \overline{\mathscr{A}}$ denoted by $\otimes\left(\mathscr{A}_{i}, x_{i}\right)$, to be the von Neumann algebra on $H$ generated by the $\mathscr{A}_{i}$.

LEMMA 3.2. (A) If $\sum\left|1-\left(x_{i} \mid y_{i}\right)\right|<\infty$ then $\otimes\left(\mathscr{A}_{i}, x_{i}\right)=\otimes\left(\mathscr{A}_{i}, y_{i}\right)$.

(B) $\otimes\left(\mathscr{A}_{i}, x_{i}\right)$ is a factor if and only if each $\mathscr{A}_{i}$ is a factor.

LEMMA 3.3. Suppose that, for each $i \in I, \mathscr{A}_{i}$ and $\mathscr{B}_{i}$ are von Neumann algebras on $H_{i}$ and $G_{i}$ respectively, that $x_{i} \in H_{i}$ and $y_{i} \in G_{i}$ with $\left\|x_{i}\right\|=\left\|y_{i}\right\|=1$, and that $\phi_{i}$ is an (algebraic) isomorphism of $\mathscr{A}_{i}$ onto $\mathscr{B}_{i}$. Suppose that $\left(\phi_{i}\left(A_{i}\right) y_{i} \mid y_{i}\right)=\left(A_{i} x_{i} \mid x_{i}\right)$ for all $A_{i} \in \mathscr{A}_{i}$ and all $i \in I$. Then there exists an isomorphism $\phi$ of $\mathscr{A}=\otimes\left(\mathscr{A}_{i}, x_{i}\right)$ onto $\mathscr{B}=\bigotimes\left(\mathscr{B}_{i}, y_{i}\right)$ which satisfies

$$
\phi\left(\bar{A}_{i}\right)=\overline{\phi_{i}\left(A_{i}\right)} \text { for all } A_{i} \in \mathscr{A}_{i} \text { and all } i \in I .
$$

Proof. This does not seem to appear explicitly in the literature; it can be proved easily, however, either by a direct proof using [2, p. 57] (the structure of isomorphisms), or by appealing to Takeda's results [11].

Lemma 3.3 enables us to make the following definition:

DefinITION 3.4. Suppose that $\left(\mathscr{A}_{i}\right)_{i \in I}$ is a family of $w^{*}$-algebras and that $\mu_{i}$ is a normal state of $\mathscr{A}_{i}$ with $\mu_{i}(1)=1$ for each $i \in I$. Suppose that $\mathscr{A}$ is a $w^{*}$-algebra and that, for each $i, \alpha_{i}$ is an isomorphism of $\mathscr{A}_{i}$ into $\mathscr{A}$. Then we will say that $\mathscr{A}$, together with $\left(\alpha_{i}\right)$, is a tensor product for the family $\left(\mathscr{A}_{i}\right)$ with respect to $\left(\mu_{i}\right)$ when the following condition is satisfied:

For every family $\left(\phi_{i}, x_{i}\right)$, where $\phi_{i}$ is a representation of $\mathscr{A}_{i}$ and $x_{i} \in S\left(\phi_{i}, \mu_{i}\right)$, there exists an isomorphism $\Lambda$ of $\mathscr{A}$ onto $\otimes\left(\phi_{i}\left(\mathscr{A}_{i}\right), x_{i}\right)$ with

$$
\Lambda\left(\alpha_{i}\left(A_{i}\right)\right)=\overline{\phi_{i}\left(A_{i}\right)}
$$

for all $A_{i} \in \mathscr{A}_{i}$ and for all $i \in I$.

Evidently the tensor product $\mathscr{A}$ of $\left(\mathscr{A}_{i}\right)$ with respect to $\left(\mu_{i}\right)$ exists and is unique up to isomorphism preserving the injections $\left(\alpha_{i}\right)$. We write $\mathscr{A}=\otimes\left(\mathscr{A}_{i}, \mu_{i}\right)$.

Definition 3.5. Suppose that $\mathscr{A}$, with canonical injections $\left(\alpha_{i}\right)$, is a tensor product for $\left(\mathscr{A}_{i}\right)$ with respect to $\left(\mu_{i}\right)$. A normal state $\nu$ of $\mathscr{A}$ will be called a product state (for the $\alpha_{i}\left(\mathscr{A}_{i}\right)$ ) if

$$
v\left(\prod_{i \in F} \alpha_{i}\left(A_{i}\right)\right)=\prod_{i \in F} \nu\left(\alpha_{i}\left(A_{i}\right)\right)
$$

for all $A_{i} \in \mathscr{A}_{i}$ and all finite subsets $F$ of $I$. 
For such a $\nu$ we write $\nu=\bigotimes \nu_{i}$ where, for each $i \in I, \nu_{i}\left(A_{i}\right)=\nu\left(\alpha_{i}\left(A_{i}\right)\right)$ for all $A_{i} \in \mathscr{A}_{i}$. Notice that, if $\otimes \nu_{i}$ exists for a family $\left(\nu_{i}\right)$, it is unique.

Lemma 3.6. Suppose that $\left(\mathscr{A}_{i}\right)_{i \in I}$ is a family of $w^{*}$-algebras, and that $\mu_{i}$ and $\nu_{i}$ are normal states of $\mathscr{A}_{i}$ with $\mu_{i}(1)=\nu_{i}(1)=1$ for each $i \in I$. Suppose that $\mathscr{A}$, with injections $\left(\alpha_{i}\right)$, is a tensor product for $\left(\mathscr{A}_{i}\right)$ with respect to $\left(\mu_{i}\right)$; and suppose that $\mathscr{B}$, with injections $\left(\beta_{i}\right)$, is a tensor product for $\left(\mathscr{A}_{i}\right)$ with respect to $\left(\nu_{i}\right)$. If

then:

$$
\sum_{i \in I}\left[1-\rho\left(\mu_{i}, \nu_{i}\right)\right]<\infty
$$

(A) $\bigotimes v_{i}$ exists on $\mathscr{A}$.

(B) There exists an isomorphism $\phi$ of $\mathscr{A}$ onto $\mathscr{B}$ such that $\phi \circ \alpha_{i}=\beta_{i}$ for all $i \in I$.

Proof. Suppose that (3.1) holds. For each $i \in I$ let $\phi_{i}$ be a representation of $\mathscr{A}_{i}$ on $H_{i}$ satisfying the conditions of Proposition 1.6. For those $i \in I$ for which $\rho\left(\mu_{i}, \nu_{i}\right) \neq 1$, we can choose $x_{i} \in S\left(\phi_{i}, \mu_{i}\right)$ and $y_{i} \in S\left(\phi_{i}, \nu_{i}\right)$ such that $\left(x_{i} \mid y_{i}\right)$ is real and

$$
1-\left(x_{i} \mid y_{i}\right)<2\left[1-\rho\left(\mu_{i}, v_{i}\right)\right] .
$$

If $\rho\left(\mu_{i}, \nu_{i}\right)=1$, we have $d\left(\mu_{i}, \nu_{i}\right)=0$ and therefore $\mu_{i}=\mu_{i}=\nu_{i}$ (Proposition 1.7), so that we can choose

$$
x_{i}=y_{i} \in S\left(\phi_{i}, \mu_{i}\right)=S\left(\phi_{i}, \nu_{i}\right) .
$$

Thus (3.2) holds for all $i \in I$, and (3.1) means that

$$
\sum_{i \in I}\left|1-\left(x_{i} \mid y_{i}\right)\right|<\infty
$$

Then $H=\bigotimes_{i \in I}\left(H_{i}, x_{i}\right)=\bigotimes_{i \in I}\left(H_{i}, y_{i}\right)$ according to Lemma 3.1. Therefore both $\mathscr{A}$ and $\mathscr{B}$ are canonically isomorphic to

$$
\bigotimes_{i \in I}\left(\phi_{i}\left(\mathscr{A}_{i}\right), x_{i}\right)=\bigotimes_{i \in I}\left(\phi_{i}\left(\mathscr{A}_{i}\right), y_{i}\right)
$$

and both (A) and (B) hold.

LEMMA 3.7. Suppose that $\left(\mathscr{A}_{i}\right)$ is a family of $w^{*}$-algebras and that $\mu_{i}$ is a normal state with $\mu_{i}(1)=1$ for each $i$. Let $\mathscr{A}=\bigotimes\left(\mathscr{A}_{i}, \mu_{i}\right)$ and let $\Sigma^{\prime}=\left\{\bigotimes \mu_{i}^{\prime}\right.$ : each $\mu_{i}^{\prime} a$ normal state of $\mathscr{A}_{i}$ and $\mu_{i}^{\prime}=\mu_{i}$ for all but a finite number of $\left.i\right\}$. Then if $A \in \mathscr{A}^{+}$and $\mu^{\prime}(A)=0$ for all $\mu^{\prime} \in \Sigma^{\prime}, A$ must be 0 .

LEMMA 3.8. If $\left(z_{i}\right)_{i \in I}$ is a family of complex numbers, $\prod_{i \in I} z_{i}$ converges if and only if $\sum_{i \in I}\left|1-z_{i}\right|<\infty$.

REMARK. See [9] for a discussion of infinite products. Recall, in particular, that convergence of a product is defined to exclude 0 as a value unless some term is 0 : a product converges if and only if the altered product obtained by deleting the 0 's converges to a nonzero number. 
4. The main results.

THEOREM 4.1. For each $i \in I$, suppose that $\mathscr{A}_{i}$ is a semifinite $w^{*}$-algebra and that $\mu_{i}$ and $\nu_{i}$ are normal states of $\mathscr{A}_{i}$ with $\mu_{i}(1)=\nu_{i}(1)=1$. Let $\mathscr{A}=\bigotimes_{i \in I}\left(\mathscr{A}_{i}, \mu_{i}\right)$. Then the following conditions on $\left(\nu_{i}\right)$ are equivalent:

(A) There exists a normal product state $\nu=\bigotimes_{i \in I} \nu_{i}$ on $\mathscr{A}$.

(B) There exists an isomorphism $\phi$ of $\mathscr{A}$ onto $\bigotimes_{i \in I}\left(\mathscr{A}_{i}, \nu_{i}\right)$ such that, for each $i \in I, \phi \circ \alpha_{i}=\beta_{i}$ where $\alpha_{i}$ is the canonical injection of $\mathscr{A}_{i}$ into $\mathscr{A}$ and $\beta_{i}$ is the canonical injection of $\mathscr{A}_{i}$ into $\bigotimes_{i \in I}\left(\mathscr{A}_{i}, v_{i}\right)$.

(C) $\sum_{i \in I}\left[d\left(\mu_{i}, \nu_{i}\right)\right]^{2}<\infty$.

(D) $\prod_{i \in I} \rho\left(\mu_{i}, v_{i}\right)$ converges.

THEOREM 4.2. For each $i \in I$, suppose that $\mathscr{A}_{i}$ and $\mathscr{B}_{i}$ are semifinite $w^{*}$-algebras, that $\mu_{i}$ is a normal state of $\mathscr{A}_{i}$ and $\nu_{i}$ is a normal state of $\mathscr{B}_{i}$ with $\mu_{i}(1)=\nu_{i}(1)=1$, and that $\phi_{i}$ is an isomorphism of $\mathscr{A}_{i}$ onto $\mathscr{B}_{i}$. Let $\mathscr{A}=\bigotimes_{i \in I}\left(\mathscr{A}_{i}, \mu_{i}\right)$ with canonical injections $\left(\alpha_{i}\right)$ and let $\mathscr{B}=\bigotimes_{i \in I}\left(\mathscr{B}_{i}, v_{i}\right)$ with canonical injections $\left(\beta_{i}\right)$. Then there exists an isomorphism $\phi$ of $\mathscr{A}$ onto $\mathscr{B}$ such that

$$
\phi\left(\alpha_{i}\left(A_{i}\right)\right)=\beta_{i}\left(\phi_{i}\left(A_{i}\right)\right)
$$

for all $A_{i} \in \mathscr{A}_{i}$ and all $i \in I$, if and only if

$$
\sum\left[d\left(\mu_{i}, \nu_{i} \circ \phi_{i}\right)\right]^{2}<\infty .
$$

TheOREM 4.3. (Cf. [1] and [7].) Suppose that, for each $i \in I, \mathscr{A}_{i}$ is a finite factor with finite normal trace $\tau_{i}$ satisfying $\tau_{i}(1)=1$. Suppose $\mu_{i}$ is a normal state of $\mathscr{A}_{i}$ with $\mu_{i}(1)=1$. Then $\mathscr{A}=\bigotimes\left(\mathscr{A}_{i}, \mu_{i}\right)$ is finite if and only if

$$
\sum\left[d\left(\mu_{i}, \tau_{i}\right)\right]^{2}<\infty
$$

Proof of 4.1. (Cf. the proof of Lemma 7 in [6].) Since $0 \leqq \rho\left(\mu_{i}, \nu_{i}\right) \leqq 1$ and $1-\rho\left(\mu_{i}, \nu_{i}\right)$ $=\frac{1}{2}\left[d\left(\mu_{i}, \nu_{i}\right)\right]^{2},(\mathrm{C})$ and (D) are equivalent (see Lemma 3.7).

To show that (A) implies (D), we will demonstrate that for (A) to hold and (D) to fail is impossible. Assume that $\nu=\bigotimes \nu_{i}$ exists on $\mathscr{A}$ and that $\prod \rho\left(\mu_{i}, \nu_{i}\right)$ diverges. The divergence of the infinite product implies that there exists a sequence $\left(F_{n}\right)_{n \in N^{+}}$ of disjoint finite subsets of $I$ such that:

$$
\prod_{i \in F_{n}} \rho\left(\mu_{i}, \nu_{i}\right)<n^{-2} \text { for all } n \in N^{+} \text {. }
$$

Let $F_{0}=I-\bigcup_{n \in N^{+}} F_{n}$. Then $\mathscr{A}$ is canonically isomorphic to $\bigotimes_{n \in N}\left(\mathscr{A}_{n}, \mu_{n}\right)$, where $\mathscr{A}_{n}=\bigotimes_{i \in F_{n}}\left(\mathscr{A}_{i}, \mu_{i}\right)$ and $\mu_{n}=\bigotimes_{i \in F_{n}} \mu_{i}$ for all $n \in N$ (the associativity of the tensor product, see [9] or [1]). Let us identify these algebras. Then $\mu=\bigotimes_{i \in I} \mu_{i}$ is identified with $\bigotimes_{n \in N} \mu_{n}$ and $\nu=\bigotimes_{i \in I} \nu_{i}$ is identified with $\bigotimes_{n \in N} \nu_{n}$. By (4.3) and the product formula for $\rho$ (Corollary 2.6), $\rho\left(\mu_{n}, \nu_{n}\right)<n^{-2}$ for all $n \in N^{+}$. Therefore (Corollary 1.13) there exists, for each $n \in N^{+}$, a projection $E_{n}$ in $\mathscr{A}_{n}$ such that

$$
\mu_{n}\left(E_{n}\right)<n^{-2} \text { and } \nu_{n}\left(E_{n}\right)>1-n^{-2} \text { for all } n \in N^{+} \text {. }
$$

Let $E=\prod_{n \in N} \gamma_{n}\left(E_{n}\right)$ where $\gamma_{n}$ is the canonical isomorphism of $\mathscr{A}_{n}$ into $\bigotimes_{n \in N}\left(\mathscr{A}_{n}, \mu_{n}\right)$ 
which has been identified with $\mathscr{A}$. Then $E$ is a projection of $\mathscr{A}$, and, using (4.4), we obtain

$$
\nu(E)=\left(\bigotimes_{n \in N} \nu_{n}\right)(E)=\prod_{n \in N^{+}} \nu_{n}\left(E_{n}\right)>\prod_{n \in N^{+}}\left(1-n^{-2}\right)>0 .
$$

Similarly $\mu(E)=0$. Now suppose $\mu^{\prime}=\bigotimes_{i \in I} \mu_{i}^{\prime}$ where $\mu_{i}^{\prime}=\mu_{i}$ for all but a finite number of $i \in I$. Then $\mu_{n}^{\prime}=\mu_{n}$ for all but a finite number of $n \in N$, and therefore, by $(4.4) \mu^{\prime}(E)=0$ for all such $\mu^{\prime}$. Hence (Lemma 3.7) $E=0$, in contradiction with (4.5). We conclude that (A) implies (D).

(D) implies (A) and (B) by Lemmas 3.6 and 3.8. Evidently (B) implies (A).

Proof of Theorem 4.2. Let $\omega_{i}=\nu_{i} \circ \phi_{i}$ so that $\omega_{i}$ is a normal state of $\mathscr{A}_{i}$. Let $\mathscr{C}=\bigotimes_{i \in I}\left(\mathscr{A}_{i}, \omega_{i}\right)$ with canonical injections $\left(\gamma_{i}\right)$. Then $\phi_{i}$ is an isormorphism of $\mathscr{A}_{i}$ onto $\mathscr{B}_{i}$ taking $\omega_{i}$ into $\nu_{i}$, and therefore there exists an isomorphism $\phi^{\prime}$ of $\mathscr{C}$ onto $\mathscr{B}$ satisfying $\phi^{\prime} \circ \gamma_{i}=\beta_{i} \circ \phi_{i}$ for all $i \in I$. Evidently, then, there exists an isomorphism $\phi$ of $\mathscr{A}$ onto $\mathscr{B}$ satisfying (4.1) if and only if there exists an isomorphism $\phi^{\prime \prime}$ of $\mathscr{A}$ onto $\mathscr{C}$ satisfying $\phi^{\prime \prime} \circ \alpha_{i}=\gamma_{i}$ for all $i \in I$. According to Theorem 4.1, that occurs if and only if

$$
\sum_{i \in I}\left[d\left(\mu_{i}, \omega_{i}\right)\right]^{2}<\infty .
$$

Proof of Theorem 4.3. $\mathscr{A}$ is necessarily a factor (Lemma 3.2).

Suppose that (4.2) holds. Then $\tau=\bigotimes \tau_{i}$ exists on $\mathscr{A}$ and can be shown to be a trace by standard arguments. Since $\mathscr{A}$ is a factor, $\tau$ is faithful. Thus $\mathscr{A}$ is finite.

To prove the necessity of (4.2), assume that $\mathscr{A}$ is finite. Then there exists a finite normal trace $\tau$ on $\mathscr{A}$ with $\tau(1)=1$. Let $\alpha_{i}$ denote the canonical injection of $\mathscr{A}_{i}$ into $\mathscr{A}=\otimes\left(\mathscr{A}_{i}, \mu_{i}\right)$. We are going to demonstrate the following formula:

$$
\tau\left(\prod_{i \in F} \alpha_{i}\left(A_{i}\right)\right)=\prod_{i \in F} \tau_{i}\left(A_{i}\right)
$$

for all $A_{i} \in \mathscr{A}_{i}$ and for all finite subsets $F$ of $I$.

From (4.6) we can conclude that $\tau=\bigotimes \tau_{i}$, and (4.2) is then a consequence of Theorem 4.1. Therefore the proof of (4.6) will complete the proof of Theorem 4.3.

Observe that (4.6) follows from the following special case:

$$
\tau\left(\prod_{i \in F} \alpha_{i}\left(E_{i}\right)\right)=\prod_{i \in F} \tau_{i}\left(E_{i}\right)
$$

for all positive integers $k_{i}$, all projections $E_{i} \in \mathscr{A}_{i}$ with $\tau_{i}\left(E_{i}\right)=2^{-k_{i}}$ and all finite subsets $F$ of $I$.

Fix the finite subset $F$ and the positive integers $k_{i}$ for $i \in F$. Let $J(i)=\left\{1,2,3, \ldots, 2^{k_{i}}\right\}$. For a projection $E_{i}$ with $\tau_{i}\left(E_{i}\right)=2^{-k_{i}}$, there exists a family $\left(E_{i}(j)\right)_{j \in J(i)}$ of projections of $\mathscr{A}_{i}$ with $E_{i}(1)=E_{i}, \sum_{j} E_{i}(j)=1$, and $\tau_{i}\left(E_{i}(j)\right)=2^{-k_{i}}$ for all $j \in J(i)$.

Given $j_{i}$ and $j_{i}^{\prime}$ in $J(i)$, there exists a unitary $U_{i} \in \mathscr{A}_{i}$ such that

$$
U_{i}\left(E_{i}\left(j_{i}\right)\right) U_{i}^{*}=E_{i}\left(j_{i}^{\prime}\right)
$$


Now let $J=\prod_{i \in F} J(i)$. For each $j=\left(j_{i}\right) \in J$, define

$$
E(j)=\prod_{i \in F} \alpha_{i}\left(E_{i}\left(j_{i}\right)\right) \text {. }
$$

Then $\sum_{j \in J} E(j)=1$. Given $j=\left(j_{i}\right)$ and $j^{\prime}=\left(j_{i}^{\prime}\right)$ in $J$, let

$$
U=\prod_{i \in F} \alpha_{i}\left(U_{i}\right)
$$

where the $U_{i}$ satisfy (4.8). Then $E\left(j^{\prime}\right)=U(E(j)) U^{*}$, and, since $\tau$ is a trace,

$$
\tau\left(E\left(j^{\prime}\right)\right)=\tau(E(j)) \text { for all } j, j^{\prime} \in J .
$$

Now $J$ has $\prod_{i \in F} 2^{k_{\mathfrak{i}}}$ elements. Therefore

$$
\tau(E(j))=\prod_{i \in F} 2^{-k_{i}} \quad \text { for all } j \in J
$$

and (4.7) follows. This completes the proof of Theorem 4.3.

\section{REFERENCES}

1. D. Bures, Certain factors constructed as infinite tensor products, Compositio Math. 15 (1963), 169-191.

2. J. Dixmier, Les algèbres d'opérateurs dans l'espace hilbertien, Gauthier-Villars, Paris, 1957.

3. - Les $C^{*}$-algèbres et leurs représentations, Gauthier-Villars, Paris, 1964.

4. H. A. Dye, The Radon-Nikodym theorem for finite rings of operators, Trans. Amer. Math. Soc. 27 (1952), 243-280.

5. A. Grothendieck, Un résultat sur le dual d'une $C^{*}$-algèbre, J. Math. Pures Appl. 36 (1957), 97-108.

6. S. Kakutani, On equivalence of infinite product measures, Ann. of Math. 49 (1948), 214-226.

7. C. C. Moore, Invariant measures on product spaces, Proc. Fifth Berkeley Sympos. Math. Stat. and Prob., 1967.

8. F. J. Murray and J. von Neumann, On rings of operators. II, Trans. Amer. Math. Soc. 41 (1937), 208-248.

9. J. von Neumann, On infinite direct products, Compositio Math. 6 (1938), 1-77.

10. S. Sakai, A Radon-Nikodym theorem in $W^{*}$-algebras, Bull. Amer. Math. Soc. 71 (1965), 149-151.

11. I. E. Segal, A non-commutative extension of abstract integration, Ann. of Math. 57 (1953), 401-457.

12. Z. Takeda, Inductive limit and infinite direct product of operator algebras, Tôhoku Math. J. 7 (1955), 67-86.

The University of British Columbia, VANCOUVER, B.C., CANADA 